\title{
The Call Option Pricing Based on Investment Strategy with Stochastic Interest Rate
}

\author{
Xin Zhang1, Huisheng Shu ${ }^{1 *}$, Xiu Kan ${ }^{2 *}$, Yingyi Fang ${ }^{1}$, Zhiwei Zheng1 \\ ${ }^{1}$ Affiliation School of Science, Donghua University, Shanghai, China \\ ${ }^{2}$ School of Electronic and Electrical Engineering, Shanghai University of Engineering Science, Shanghai, China \\ Email: *hsshu@dhu.edu.cn, ${ }^{\star x}$ xiu.kan@sues.edu.cn
}

How to cite this paper: Zhang, X., Shu, H.S., Kan, X., Fang, Y.Y. and Zheng, Z.W. (2018) The Call Option Pricing Based on Investment Strategy with Stochastic Interest Rate. Journal of Mathematical Finance, 8, 43-57.

https://doi.org/10.4236/jmf.2018.81004

Received: October 27, 2017

Accepted: January 26, 2018

Published: January 29, 2018

Copyright (ㅇ 2018 by authors and Scientific Research Publishing Inc. This work is licensed under the Creative Commons Attribution International License (CC BY 4.0).

http://creativecommons.org/licenses/by/4.0/

\begin{abstract}
In this paper, the call option price is evaluated based on linear investment strategy in order to hedge the risk actively in stock market with stochastic interest rate. The Vasicek model is used to describe the structure of interest rates. The mathematical characterization is discussed for the unique no-arbitrage price associated with any attainable contingent claim. The appropriate numeraire (zero-coupon bond) and measures ( $T$-forward measure) are chosen to simplify the calculations. Based on the designed linear investment strategy with stochastic interest rate, a novel option price approach is obtained under the $T$-forward measure.
\end{abstract}

\section{Keywords}

Europe Call Option, Investment Strategy, Stochastic Interest Rate, Vasicek Model, Numeraire and Measures, $T$-Forward Measure

\section{Introduction}

With the globalization of economy and the rapid development of financial derivatives market, the analysis of the option pricing is of great importance and has attracted considerable attention. In 1973, Black and Scholes [1] proposed the classical Black-Scholes model under the risk-neutral world with the assumption that the stock price follows geometric Brownian motion, and the expected return rate stock is a constant, while, the assumption of Black-Scholes model has a large difference with the real world. Merton [2] considered dividend and stochastic interest rate into the option pricing model. Cox and Rose [3] [4] used the alternative stochastic process to discuss the option pricing model and considered the expanded formula of stock price that does not include continuous sample path. 
Buraschi and Dumas [5] studied the option pricing which the underlying assets obey to general diffusion process, deduced analytical formula and forward integral expressed based on the boundary of Europe option pricing. However, the implied assumption, throughout the traditional option pricing model, is that the investors do not have any stock trading at all during the valid period. Indeed, the investors could carry out reasonable stock trading strategy to guard against risks caused by the change of stock price. Wang [6] innovatively proposed the stock option pricing model based on dynamic investment strategy. In this case, the investors could purchase stock consequently with the stock price rising continuously while the stock price is higher than the call option striking price. This stock investment strategy could help investors reduce some risk. Wang [7] considered the several linear investment strategies into the stock option price as well, and deduced an option pricing model formula through the complex mathematical calculation, while, Yan [8] calculated the option pricing model formula under the nonlinear dynamic investment strategy.

It is known to all that the classical Black-Scholes model presumes the interest rate is a constant. In real financial market, however, the rate is fluctuating generally influenced by national policy, economy and stock market. In this case, many academic proposed stochastic interest rate model to research the option pricing issue. Generally speaking, the research includes the interest rate term structure, correction and expansion based on Black-Scholes model. In 1977, Vasicek [9] by using the basic Black-Scholes argument, developed a model that considers the evolution of the term structure of interest rates and the pricing of interest-rate derivatives, and the Vasicek model exhibits mean reversion characteristic. Harrison \& Kreps [10] and Harrison \& Pliska [11] [12] developed the first rigorous approach for the arbitrage-free pricing of general contingent claims. Jarrow and Morton [13] developed a general framework for interest-rates dynamics. Geman et al. [14] provided a useful toolkit explaining how the various dynamics change when changing the numeraire, where the apposite choice of numeraire could simplify the option calculations. Musiela and Rutkowski [15] and Hunt and Kennedy [16] gave more details about the treatment of the no-arbitrage issue under stochastic interest rates. Jamshidian [17] first used the change of measure in the calculation of a bond-option price under the Vasicek model, the approach of which provide the method to calculate the stock option price under the change of measure. Wang and Zhang [18] presented Europe call geometric option when the stochastic interest rate is modeled by Vasicek model and Hull-White model. Yao and Wang [19] used geometric mean to calculate average price of the asset and obtained average price, average strike price option pricing formula and parity formula under the continuous time situation. Xiao [20] gave the solution of Black-Scholes formula under the partial differential equation method, the equivalent martingale measure method, and the derivation of European option pricing with the stochastic interest rate, paid dividend and jump-diffusion. Feng [21] analyzed and simulated the Vasicek interest rate 
model and MHL interest rate model, observed the option error, and concluded that under the Black-Scholes model, MHL model in the medium-term and short-term index call option pricing errors smaller than in the short-term Vasicek interest rate model index call option pricing error. Cui [22] discussed the correlation of two Brownian motions which are in the Hull-White model the interest rate and Black-Scholes model the underlying assets, under the assumption of stochastic interest rates, the simulation methods which are similar to the classic binary tree.

As we all known, the option pricing issue under the stochastic interest rate model has been extensive researched, but the option pricing under investment strategy is still a new filed. Investors draw up the stock investment strategy while purchases stock call option. During the option valid period, investors can purchase stock according to the fixed investment strategy which is linear correlated with the stock price. Meanwhile, the investment strategy is taken when the stock price higher than the striking price. Under this circumstances, the loss of position stock with trading planned stock will less than that not trading, the new option price under the designed investment strategy could be less than the classical Black-Scholes model option price. Wang [6] has proved this result correctly but under the assumption of the interest rate is a constant. In this paper, the interest rate term structure will be considered into the new option price model under the designed investment strategy. A favorable numeraire, zero-coupon bond, is chosen through the martingale measure theorem. Furthermore, the new stock option can be achieved by calculating an expectation of its payoff which calculated under the designed investment strategy.

\section{Prerequisite Knowledge}

In this paper, we consider two assets in the market. One is the riskiness asset, the savings account $B_{t}$ with dynamics

$$
B_{t}=\mathrm{e}^{\int_{0}^{t} r_{s} \mathrm{~d} s}
$$

or in difference form

$$
\mathrm{d} B_{t}=r_{t} B_{t} \mathrm{~d} t
$$

where $r_{t}$ is the named risk-free interest rate at time $t$. The other is the risky asset supposed to be the stock. Consider the widely used model of stock price as follow

$$
\mathrm{d} S_{t}=\mu_{t} S_{t} \mathrm{~d} t+\sigma_{1} S_{t} \mathrm{~d} W_{1, t}
$$

where $\mu_{t}$ is the expected rate of return of the stock price at time $t$. Under the risk-neutral world, the $\mu_{t}$ is equivalent to risk-free interest rate $r_{t}, \sigma_{1}$ assumed constant, is usually referred to as the stock price volatility, $W_{t}^{1}$ is a normal Brownian motion. Thus, the stock price, under the risk-neutral world, could be given by

$$
\mathrm{d} S_{t}=r_{t} S_{t} \mathrm{~d} t+\sigma_{1} S_{t} \mathrm{~d} W_{1, t} .
$$


From the Itô formula, the stock price at time $T$ can be expressed as

$$
S_{T}=S_{0} \mathrm{e}^{\int_{0}^{T}\left(r_{\mathrm{s}}-\frac{\sigma_{1}^{2}}{2}\right) \mathrm{d} s+\int_{0}^{T} \sigma_{1} \mathrm{~d} W_{1, t}}
$$

with $S_{0}$ is the stock price at initial time.

$\left(\Omega, \mathcal{F},\left\{\mathcal{F}_{t}\right\}_{t \geq 0}, P\right)$ is a complete probability space with the filtration $\left\{\mathcal{F}_{t}\right\}_{t \geq 0}$ follows the usual conditions. $[0, T]$ is the time interval where 0 and $T$ respectively represents current time and the expiry date. In this paper, we are interest in calculating the new option price under stochastic interest rates. While, the existence of the stochastic factors complicates the calculation. In such cases, considering a change of measure and numeraire would be quite helpful.

Definition 2.1. [14] A numeraire is any positive non-dividend-paying asset.

Remark 2.1. Intuitively, a numeraire is a reference asset that is chosen so as to normalize all other asset prices with respect to it. In fact, each numeraire can be represented as a stochastic process and corresponding a probability measure. The risk-neutral numeraire is the money-market account defined in equation (2.1) and (2.2).

Lemma 2.1. [14] Assume there exists a numeraire $N$ and corresponding probability measure $Q_{N}$, such that the price of any traded asset (without intermediate payments) $X$ relative to $N$ is a martingale under $Q_{N}$, i.e.

$$
\frac{X_{t}}{N_{t}}=E^{N}\left[\frac{X_{T}}{N_{T}} \mid \mathcal{F}_{t}\right], 0 \leq t \leq T .
$$

In many concrete situations, a useful numeraire is the zero-coupon bond $P(t, T)$ maturing at time $T$ where $0 \leq t \leq T$ [17]. The measure associated with this numeraire is referred to as $T$-forward risk-adjusted measure, or more briefly as $T$-forward measure, and denoted $Q^{T}$. The related expectation is denoted by $E^{T}$, in such case, $P(T, T)=1$. Thus, pricing the derivative $H_{0}$ at initial time can be achieved by calculating an expectation of its payoff

$$
H_{0}=P(0, T) E^{T}\left[H_{T} \mid \mathcal{F}_{0}\right]
$$

where $H_{T}$ is the claim payoff at time $T$. We know that the filtration $\mathcal{F}_{0}$ has no influence on the above calculation of the expectation. This means that the formula can also been written as follows:

$$
H_{0}=P(0, T) E^{T}\left[H_{T}\right] .
$$

Indeed, according to risk-neutral argument, the zero-coupon bond price at initial time can be given by

$$
P(0, T)=E\left[\mathrm{e}^{-\int_{0}^{T} r_{\mathrm{s}} \mathrm{d} s}\right]
$$

with $E$ denoting the expectation under the risk-neutral measure.

Then the option price $C$ at initial time on a zero coupon bond under the $T$ forward measure could be calculated by

$$
C=P(0, T) E^{T}\left[V_{T}\right]
$$


where $V_{T}$ is the claim payoff of the option at time $T$.

\section{The Europe Call Option Price Based on Investment Strategy}

In this section, we shall discuss the new option price, the Europe call option price, based on investment strategy with the stochastic interest rates under the Vasicek short model. Now, we deduce the intrinsic value function about the above new option. Consider that the Europe call option under the linear stock investment strategy that the initiatory buying price is higher than the striking price. The investment percentage function $Q(S)$ is given as follows [6]:

$$
Q(S)= \begin{cases}0 & S \leq K, \\ \frac{\beta}{\alpha K}(S-K) & K \leq S \leq(1+\alpha) K, \\ \beta & S \geq(1+\alpha) K\end{cases}
$$

where

$S$ is stock price;

$A$ is all investment amount;

$Q(S)$ is the stock investment proportion, which is equal to the value of the stock investment divided by $A$;

$K$ is strike price of the option;

$\alpha$ is investment strategy index, whereby the stock investment occurs while the stock price varies from $K$ to $(1+\alpha) K$, the smaller $\alpha$ indicates that the investors buy stock in a narrower interval of stock prices;

$\beta$ is the maximum value of the stock investment proportion.

The feature of function $Q(S)$ is shown in Figure 1, from which we can easily see that the added investment is $\Delta q$ when the stock pricing changes from $S-\Delta S$ to $S$, and

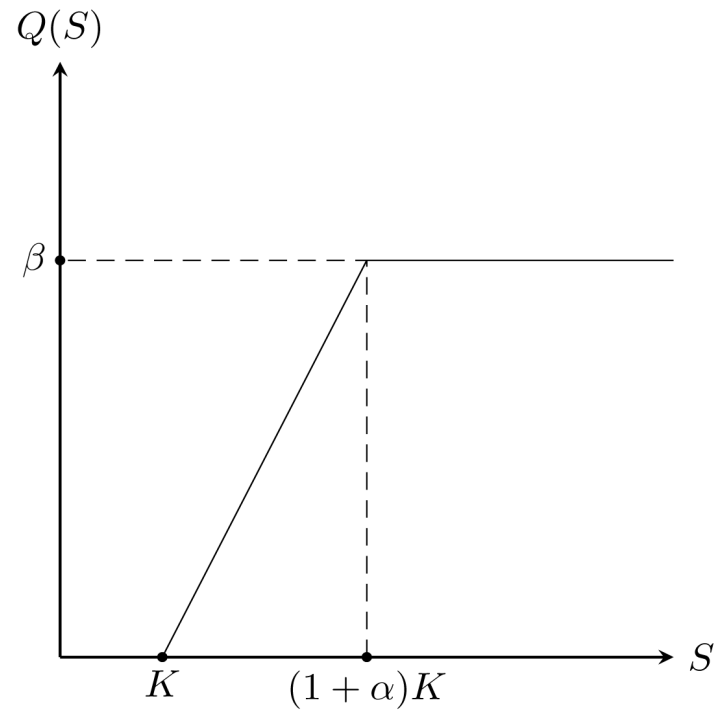

Figure 1. Feature of function $Q(S)$. 


$$
\Delta q=\Delta Q A=\Delta S Q^{\prime}(S) A
$$

Thus, the increased share of investment stock is $\frac{\Delta q}{S}$. If the stock price rises to $S_{0}$, the income of this investment $\Delta q$ should be given by

$$
\frac{\Delta q}{S}\left(S_{0}-S\right)
$$

Therefore, while the price varies from $K$ to $S_{0}$, the income $R\left(S_{0}\right)$, under the above stock investment strategy, can be expressed by integral form as follows:

$$
R\left(S_{0}\right)=\int_{K}^{S_{0}} \frac{A Q^{\prime}(S)}{S}\left(S_{0}-S\right) \mathrm{d} S .
$$

Notice that $Q^{\prime}(S)=0$ when $S \geq(1+\alpha) K$, then, the integration can be represented by

$$
R\left(S_{0}\right)= \begin{cases}\int_{K}^{S_{0}} \frac{A Q^{\prime}(S)}{S}\left(S_{0}-S\right) \mathrm{d} S & K \leq S_{0} \leq(1+\alpha) K, \\ \int_{K}^{(1+\alpha) K} \frac{A Q^{\prime}(S)}{S}\left(S_{0}-S\right) \mathrm{d} S & S_{0}>(1+\alpha) K .\end{cases}
$$

Calculating the above integral formula by the derivative of $Q(S)$, we can obtain the income function at stock price $S_{0}$ as follows:

$$
R\left(S_{0}\right)= \begin{cases}\frac{A \beta S_{0}}{\alpha K} \ln \left(\frac{S_{0}}{K}\right)-\frac{A \beta}{\alpha K}\left(S_{0}-K\right) & K \leq S_{0} \leq(1+\alpha) K, \\ \frac{A \beta S_{0}}{\alpha K} \ln (1+\alpha)-A \beta & S_{0}>(1+\alpha) K .\end{cases}
$$

If the stock price varies from $K$ to $S_{0}$, the investors do not buy any stock. Under this condition, the short position's expected loss is given by

$$
\frac{A}{K}\left(S_{0}-K\right)
$$

In this case, however, the holding stock by the investor based on the above investment strategy will offset some loss, and the loss function would be

$$
\frac{A}{K}\left(S_{0}-K\right)-R\left(S_{0}\right)
$$

divided by $A / K$, the loss function $L\left(S_{0}\right)$ of each share is

$$
L\left(S_{0}\right)= \begin{cases}\left(1+\frac{\beta}{\alpha}\right)\left(S_{0}-K\right)-\frac{\beta S_{0}}{\alpha} \ln \left(\frac{S_{0}}{K}\right) & K \leq S_{0} \leq(1+\alpha) K, \\ S_{0}-K-\frac{\beta S_{0}}{\alpha} \ln (1+\alpha)+K \beta & S_{0}>(1+\alpha) K .\end{cases}
$$

As known to all, the option seller should compensate the expected loss of the option holder in option market. Therefore, the value of loss function is equivalent to the claim payoff. Noticed that the option claim payoff is zero when $S_{T} \leq K$, so that the claim payoff of the new option $V_{T}$ at time $T$ can be expressed as 


$$
V_{T}= \begin{cases}0 & S_{T} \leq K \\ \left(1+\frac{\beta}{\alpha}\right)\left(S_{T}-K\right)-\frac{\beta S_{T}}{\alpha} \ln \left(\frac{S_{T}}{K}\right) & K \leq S_{T} \leq(1+\alpha) K, \\ S_{T}-K-\frac{\beta S_{T}}{\alpha} \ln (1+\alpha)+K \beta & S_{T}>(1+\alpha) K\end{cases}
$$

where $S_{T}$ is the stock price at maturity time $T$.

Many options are priced of constant interest rate under the assumption, which we can see in Black-Scholes model. However, in financial markets, the interest rate cannot be assumed as an unchanging factor. Here, we use classical short-rate model, the Vasicek model, to evaluate the new option price.

Vasicek (1977) assumed that the instantaneous spot rate, under the real-world measure, evolves as an Ornstein-Uhlenbeck process with constant modulus. For a suitable choice of the market price of risk, this dynamics coincide with the other dynamics as well, which the short rate follows an Ornstein-Uhlenbeck process with constant coefficients under the risk-neutral measure [23], that is

$$
\mathrm{d} r_{t}=\left(\theta-a r_{t}\right) \mathrm{d} t+\sigma_{2} \mathrm{~d} W_{2, t}
$$

which can also be expressed as

$$
\mathrm{d} r_{t}=a\left(b-r_{t}\right) \mathrm{d} t+\sigma_{2} \mathrm{~d} W_{2, t}
$$

where

$r_{t}$ is the short rate at time $t$;

$a$ is the speed of mean reversion;

$b$ is mean reversion level, and $\theta=a b$;

$\sigma_{2}$ is the short rate volatility;

$W_{2, t}$ is normal Brownian Motion process at time $t$ and independent with $W_{1, t}$.

The Vasicek models drift term means that over time interest rates will converge to the mean reversion level $b$ with the speed $a$. The parameter $b$ can be thought as the long interest rate level, that is the process swing around the level $b$ in an erratic but continuous.

Collecting $r_{t}$ terms we can obtain

$$
r_{t}=\mathrm{e}^{-a t} r_{0}+\frac{\theta}{a}\left(1-\mathrm{e}^{-a t}\right)+\sigma_{2} \int_{0}^{t} \mathrm{e}^{-a(t-u)} \mathrm{d} W_{2, t}
$$

where $r_{0}$ is the short rate at time 0 . Then, the short rate $r_{t}$ is normally distributed with mean and variance given respectively by

$$
\begin{aligned}
& E\left[r_{t}\right]=\mathrm{e}^{-a t} r_{0}+\frac{\theta}{a}\left(1-\mathrm{e}^{-a t}\right), \\
& \operatorname{Var}\left[r_{t}\right]=\frac{\sigma_{2}^{2}}{2 a}\left(1-\mathrm{e}^{-2 a t}\right) .
\end{aligned}
$$

Meanwhile, the price of zero coupon bond can be evaluated by the expectation of a functional of the process $r_{t}$ as

$$
P(0, T)=E\left[\mathrm{e}^{-\int_{0}^{T} r_{u} \mathrm{~d} u}\right]=\mathrm{e}^{E\left[-\int_{0}^{T} r_{u} \mathrm{~d} u\right]+\frac{1}{2} \operatorname{Var}\left[-\int_{0}^{T} r_{u} \mathrm{~d} u\right]}=A(0, T) \mathrm{e}^{-r_{0} B(0, T)}
$$


where

$$
\begin{aligned}
& A(0, T)=\exp \left\{[B(0, T)-T]\left(\frac{\theta}{a}-\frac{\sigma_{2}^{2}}{2 a^{2}}\right)-\frac{\sigma_{2}^{2} B(0, T)^{2}}{4 a}\right\}, \\
& B(0, T)=\frac{1-\mathrm{e}^{-a T}}{a} .
\end{aligned}
$$

The price of zero coupon bond have been computed by instantaneous-spotrate dynamics under the risk-neutral measure. In order to evaluate the new option price, we only need to calculate the expectation of $V_{T}$ under the T-forward measure. The change of measure and the Formula (3.10) imply that under the $T$-forward measure $Q^{T}$ (has been derived by Jamshidian [17])

$$
\mathrm{d} r_{t}=\left(\theta-a r_{t}-\sigma_{2}^{2} B(t, T)\right) \mathrm{d} t+\sigma_{2} \mathrm{~d} W_{2, t}^{T}
$$

where the $Q^{T}$-Brownian motion $W_{2, t}^{T}$ is defined by

$$
\mathrm{d} W_{2, t}^{T}=\mathrm{d} W_{2, t}+\sigma_{2}^{2} B(t, T) \mathrm{d} t,
$$

and

$$
B(t, T)=\frac{1-\mathrm{e}^{-a(T-t)}}{a} .
$$

Thus,

$$
r_{t}=\mathrm{e}^{-a t} r_{0}+M^{T}(0, t)+\sigma_{2} \int_{0}^{t} \mathrm{e}^{-a(t-u)} \mathrm{d} W_{2, u}^{T}
$$

with

$$
M^{T}(0, t)=\left(\frac{\theta}{a}-\frac{\sigma_{2}^{2}}{a^{2}}\right)\left(1-\mathrm{e}^{-a t}\right)+\frac{\sigma_{2}^{2}}{2 a^{2}}\left(\mathrm{e}^{-a(T-t)}-\mathrm{e}^{-a(T+t)}\right) .
$$

Evaluating the integral of the short rate $r_{t}$ on $(0, T)$

$$
\begin{aligned}
\int_{0}^{T} r_{t} \mathrm{~d} t= & \int_{0}^{T} \mathrm{e}^{-a t} r_{0} \mathrm{~d} t+\int_{0}^{T} M^{T}(0, t) \mathrm{d} t+\int_{0}^{T} \sigma_{2} \int_{0}^{t} \mathrm{e}^{-a(t-u)} \mathrm{d} W_{2, u}^{T} \mathrm{~d} t \\
= & r_{0} \frac{1-\mathrm{e}^{-a T}}{a}+\left(\frac{\theta}{a}-\frac{\sigma_{2}^{2}}{a^{2}}\right)\left(T+\frac{\mathrm{e}^{-a T}-1}{a}\right) \\
& +\frac{\sigma_{2}^{2}}{2 a^{2}}\left(\frac{1-2 \mathrm{e}^{-a T}+\mathrm{e}^{-2 a T}}{a}\right) \\
& +\sigma_{2} \int_{0}^{T} \int_{0}^{t} \mathrm{e}^{-a(t-u)} \mathrm{d} W_{2, u}^{T} \mathrm{~d} t
\end{aligned}
$$

As the same analysis, the dynamic of the process $S_{t}$ under the T-forward measure can be expressed by

$$
\mathrm{d} S_{t}=r_{t} S_{t} \mathrm{~d} t+\sigma_{1} S_{t} \mathrm{~d} W_{1, t}^{T} .
$$

The Girsanov theorem implies that the process $W_{1, t}^{T}$ defined by [23]

$$
W_{1, t}^{T}=W_{1, t}
$$

Now we calculate the expectation of $V_{T}$ under the T-forward measure. It is important to note that the random variable $S_{T}$ is lognormally distributed, $f\left(S_{T}\right)$ denotes the probability density function of $S_{T}$, we can obtain that 


$$
\begin{aligned}
E^{T}\left[V_{T}\right]= & \int_{K}^{(1+\alpha) K}\left[\left(1+\frac{\beta}{\alpha}\right)\left(S_{T}-K\right)-\frac{\beta S_{T}}{\alpha} \ln \frac{S_{T}}{K}\right] f\left(S_{T}\right) \mathrm{d} S_{T} \\
& +\int_{(1+\alpha) K}^{+\infty}\left[S_{T}-K-\frac{\beta S_{T}}{\alpha} \ln (1+\alpha)+K \beta\right] f\left(S_{T}\right) \mathrm{d} S_{T} \\
= & \int_{K}^{(1+\alpha) K}\left(1+\frac{\beta}{\alpha}\right)\left(S_{T}-K\right) f\left(S_{T}\right) \mathrm{d} S_{T}-\int_{K}^{(1+\alpha) K} \frac{\beta S_{T}}{\alpha} \ln \frac{S_{T}}{K} f\left(S_{T}\right) \mathrm{d} S_{T} \\
& +\int_{(1+\alpha) K}^{+\infty}\left[\left(1-\frac{\beta}{\alpha} \ln (1+\alpha)\right) S_{T}+K(\beta-1)\right] f\left(S_{T}\right) \mathrm{d} S_{T} .
\end{aligned}
$$

In order to simplify the process of integral calculation, we divide the above expectation into three parts given by

$$
\begin{aligned}
& I_{1}=\int_{K}^{(1+\alpha) K}\left(1+\frac{\beta}{\alpha}\right)\left(S_{T}-K\right) f\left(S_{T}\right) \mathrm{d} S_{T}, \\
& I_{2}=-\int_{K}^{(1+\alpha) K} \frac{\beta S_{T}}{\alpha} \ln \frac{S_{T}}{K} f\left(S_{T}\right) \mathrm{d} S_{T}, \\
& I_{3}=\int_{(1+\alpha) K}^{+\infty}\left[\left(1-\frac{\beta}{\alpha} \ln (1+\alpha)\right) S_{T}+K(\beta-1)\right] f\left(S_{T}\right) \mathrm{d} S_{T} .
\end{aligned}
$$

LET $y=\ln S_{T}$, it is known from probability theory that $f\left(\mathrm{e}^{y}\right) \mathrm{e}^{y}$ is the probability density function of $\ln S_{T}$, whose mean and variance under the $T$-forward measure can be expressed from (2.5) respectively as follows:

$$
\begin{aligned}
\mu_{T}= & E^{T}\left[\ln S_{T}\right]=E^{T}\left[\ln S_{0}+\int_{0}^{T} r_{t}-\frac{\sigma_{1}^{2}}{2} \mathrm{~d} t+\int_{0}^{T} \sigma_{1} \mathrm{~d} W_{1, t}^{T}\right] \\
= & \ln S_{0}-\frac{\sigma_{1}^{2}}{2} T+E^{T}\left[\int_{0}^{T} r_{t} \mathrm{~d} t\right] \\
= & \ln S_{0}-\frac{\sigma_{1}^{2}}{2} T-r_{0} \frac{1-\mathrm{e}^{-a T}}{a}+\left(\frac{\theta}{a}-\frac{\sigma_{2}^{2}}{a^{2}}\right)\left(T+\frac{\mathrm{e}^{-a T-1}}{a}\right) \\
& +\frac{\sigma_{2}^{2}}{2 a^{2}} \frac{1-2 \mathrm{e}^{-a T}+\mathrm{e}^{-2 a T}}{a} \\
= & \ln S_{0}-\frac{\sigma_{1}^{2}}{2} T-r_{0} B(0, T)+\left(\frac{\theta}{a}-\frac{\sigma_{2}^{2}}{a^{2}}\right)[T-B(0, T)]+\frac{\sigma_{2}^{2}}{2 a} B(0, T)^{2}, \\
\sigma_{T}^{2}= & \operatorname{Var}^{T}\left[\ln S_{0}+\int_{0}^{T} r_{t} \mathrm{~d} t-\int_{0}^{T} \frac{\sigma_{1}^{2}}{2} \mathrm{~d} t+\int_{0}^{T} \sigma_{1} \mathrm{~d} W_{1, t}^{T}\right] \\
= & \operatorname{Var}^{T}\left[\sigma_{2} \int_{0}^{T} \int_{0}^{t} \mathrm{e}^{-a(t-u)} \mathrm{d} W_{u}^{T} \mathrm{~d} t+\int_{0}^{T} \sigma_{1} \mathrm{~d} W_{1, t}^{T}\right] \\
= & \frac{\sigma_{2}^{2}}{a^{2}}\left[T-2 \frac{1-\mathrm{e}^{-a T}}{a}+\frac{1-\mathrm{e}^{-2 a T}}{2 a}\right]+\sigma_{1}^{2} T \\
= & \frac{\sigma_{2}^{2}}{a^{2}}\left[T-2 B(0, T)+\frac{1-\mathrm{e}^{-2 a T}}{2 a}\right]+\sigma_{1}^{2} T,
\end{aligned}
$$

moreover, we have

$$
f\left(\mathrm{e}^{y}\right) \mathrm{e}^{y}=\frac{1}{\sqrt{2 \pi} \sigma_{T}} \mathrm{e}^{-\frac{1\left(y-\mu_{T}\right)^{2}}{2} \sigma_{T}^{2}} .
$$


Thus,

$$
\begin{aligned}
I_{1}= & \int_{K}^{(1+\alpha) K}\left(1+\frac{\beta}{\alpha}\right)\left(S_{T}-K\right) f\left(S_{T}\right) \mathrm{d} S_{T} \\
= & \left(1+\frac{\beta}{\alpha}\right) \int_{\ln K}^{\ln (1+\alpha) K}\left(\mathrm{e}^{y}-K\right) f\left(\mathrm{e}^{y}\right) \mathrm{e}^{y} \mathrm{~d} y \\
= & \left(1+\frac{\beta}{\alpha}\right) \frac{1}{\sqrt{2 \pi} \sigma_{T}} \int_{\ln K}^{\ln (1+\alpha) K} \mathrm{e}^{y} \mathrm{e}^{-\frac{1}{2} \frac{\left(y-\mu_{T}\right)^{2}}{\sigma_{T}^{2}}} \mathrm{~d} y \\
& -K\left(1+\frac{\beta}{\alpha}\right) \frac{1}{\sqrt{2 \pi} \sigma_{T}} \int_{\ln K}^{\ln (1+\alpha) K} \mathrm{e}^{-\frac{1}{2} \frac{\left(y-\mu_{T}\right)^{2}}{\sigma_{T}^{2}}} \mathrm{~d} y .
\end{aligned}
$$

Define

$$
z=\frac{y-\mu_{T}}{\sigma_{T}}
$$

or equivalently

$$
y=\mu_{T}+z \sigma_{T} .
$$

Then,

$$
\begin{aligned}
I_{1}= & \left(1+\frac{\beta}{\alpha}\right) \frac{1}{\sqrt{2 \pi}} \int_{\frac{\ln K-\mu_{T}}{\sigma_{T}}}^{\frac{\ln (1+\alpha) K-\mu_{T}}{\sigma_{T}}} \mathrm{e}^{\mu_{T}+\sigma_{T} z} \mathrm{e}^{-\frac{1}{2} z^{2}} \mathrm{~d} z-K\left(1+\frac{\beta}{\alpha}\right) \frac{1}{\sqrt{2 \pi}} \int_{\frac{\ln K-\mu_{T}}{\sigma_{T}}}^{\frac{\ln (1+\alpha) K-\mu_{T}}{\sigma_{T}}} \mathrm{e}^{-\frac{1}{2} z^{2}} \mathrm{~d} z \\
= & \left(1+\frac{\beta}{\alpha}\right) \frac{1}{\sqrt{2 \pi}} \int_{\frac{\ln K-\mu_{T}}{\sigma_{T}}}^{\frac{\ln (1+\alpha) K-\mu_{T}}{\sigma_{T}}} \mathrm{e}^{\mu_{T}+\frac{1}{2} \sigma_{T}^{2}} \mathrm{e}^{-\frac{1}{2}\left(z-\sigma_{T}\right)^{2}} \mathrm{~d} z-K\left(1+\frac{\beta}{\alpha}\right) \frac{1}{\sqrt{2 \pi}} \int_{\frac{\ln K-\mu_{T}}{\sigma_{T}}}^{\frac{\ln (1+\alpha) K-\mu_{T}}{\sigma_{T}}} \mathrm{e}^{-\frac{1}{2} z^{2}} \mathrm{~d} z \\
= & \left(1+\frac{\beta}{\alpha}\right) \mathrm{e}^{\mu_{T}+\frac{1}{2} \sigma_{T}^{2}}\left[N\left(\frac{\ln (1+\alpha) K-\mu_{T}-\sigma_{T}^{2}}{\sigma_{T}}\right)-N\left(\frac{\ln K-\mu_{T}-\sigma_{T}^{2}}{\sigma_{T}}\right)\right] \\
& -K\left(1+\frac{\beta}{\alpha}\right)\left[N\left(\frac{\ln (1+\alpha) K-\mu_{T}}{\sigma_{T}}\right)-N\left(\frac{\ln K-\mu_{T}}{\sigma_{T}}\right)\right]
\end{aligned}
$$

where $N(x)$ is the standard normal cumulative density function, simplify the above integration

$$
I_{1}=\left(1+\frac{\beta}{\alpha}\right) \mathrm{e}^{\mu_{T}+\frac{1}{2} \sigma_{T}^{2}}\left[N\left(d_{1}\right)-N\left(d_{2}\right)\right]-K\left(1+\frac{\beta}{\alpha}\right)\left[N\left(d_{3}\right)-N\left(d_{4}\right)\right]
$$

with

$$
\begin{aligned}
& d_{1}=\frac{\ln (1+\alpha) K-\mu_{T}-\sigma_{T}^{2}}{\sigma_{T}}, \\
& d_{2}=\frac{\ln K-\mu_{T}-\sigma_{T}^{2}}{\sigma_{T}}, \\
& d_{3}=\frac{\ln (1+\alpha) K-\mu_{T}}{\sigma_{T}}, \\
& d_{4}=\frac{\ln K-\mu_{T}}{\sigma_{T}} .
\end{aligned}
$$

Similarly, we have 


$$
\begin{aligned}
& I_{2}=-\int_{K}^{(1+\alpha) K} \frac{\beta S_{T}}{\alpha} \ln \frac{S_{T}}{K} f\left(S_{T}\right) \mathrm{d} S_{T} \\
& =-\int_{K}^{(1+\alpha) K} \frac{\beta S_{T}}{\alpha} \ln S_{T} f\left(S_{T}\right) \mathrm{d} S_{T}+\int_{K}^{(1+\alpha) K} \frac{\beta S_{T}}{\alpha} \ln K f\left(S_{T}\right) \mathrm{d} S_{T} \\
& =-\frac{\beta}{\alpha} \int_{\ln K}^{\ln (1+\alpha) K} y \mathrm{e}^{y} f\left(\mathrm{e}^{y}\right) \mathrm{e}^{y} \mathrm{~d} y+\frac{\beta}{\alpha} \int_{\ln K}^{\ln (1+\alpha) K} \ln K \mathrm{e}^{y} f\left(\mathrm{e}^{y}\right) \mathrm{e}^{y} \mathrm{~d} y \\
& =-\frac{\beta}{\alpha} \frac{1}{\sqrt{2 \pi} \sigma_{T}} \int_{\ln K}^{\ln (1+\alpha) K} y \mathrm{e}^{y} \mathrm{e}^{-\frac{1}{2} \frac{\left(y-\mu_{T}\right)^{2}}{\sigma_{T}^{2}}} \mathrm{~d} y+\frac{\beta}{\alpha} \frac{1}{\sqrt{2 \pi} \sigma_{T}} \int_{\ln K}^{\ln (1+\alpha) K} \ln K \mathrm{e}^{y} \mathrm{e}^{-\frac{1}{2} \frac{\left(y-\mu_{T}\right)^{2}}{\sigma_{T}^{2}}} \mathrm{~d} y \\
& =-\frac{\beta}{\alpha} \frac{1}{\sqrt{2 \pi}} \int_{\frac{\ln K-\mu_{T}}{\sigma_{T}}}^{\frac{\ln (1+\alpha) K-\mu_{T}}{\sigma_{T}}}\left(\mu_{T}+z \sigma_{T}\right) \mathrm{e}^{\mu_{T}+z \sigma_{T}} \mathrm{e}^{-\frac{1}{2} z^{2}} \mathrm{~d} z \\
& +\frac{\beta}{\alpha} \frac{1}{\sqrt{2 \pi}} \int_{\frac{\ln K-\mu_{T}}{\sigma_{T}}}^{\frac{\ln (1+\alpha) K-\mu_{T}}{\sigma_{T}}} \ln K \mathrm{e}^{\mu_{T}+\sigma_{T} z} \mathrm{e}^{-\frac{1}{2} z^{2}} \mathrm{~d} z \\
& =-\frac{\beta}{\alpha}\left(\mu_{T}-\ln K\right) \mathrm{e}^{\mu_{T}+\frac{1}{2} \sigma_{T}^{2}}\left[N\left(\frac{\ln (1+\alpha) K-\mu_{T}-\sigma_{T}^{2}}{\sigma_{T}}\right)-N\left(\frac{\ln K-\mu_{T}-\sigma_{T}^{2}}{\sigma_{T}}\right)\right] \\
& -\frac{\beta}{\alpha} \frac{1}{\sqrt{2 \pi}} \int_{\frac{\ln K-\mu_{T}}{\sigma_{T}}}^{\frac{\ln (1+\alpha) K-\mu_{T}}{\sigma_{T}}} \sigma_{T} z \mathrm{e}^{\mu_{T}+\sigma_{T} z} \mathrm{e}^{-\frac{1}{2} z^{2}} \mathrm{~d} z \\
& =-\frac{\beta}{\alpha}\left(\mu_{T}-\ln K\right) \mathrm{e}^{\mu_{T}+\frac{1}{2} \sigma_{T}^{2}}\left[N\left(d_{1}\right)-N\left(d_{2}\right)\right] \\
& -\frac{\beta}{\alpha} \frac{\sigma_{T}}{\sqrt{2 \pi}} \mathrm{e}^{\mu_{T}+\frac{1}{2} \sigma_{T}^{2}}\left(\mathrm{e}^{-\frac{d_{2}^{2}}{2}}-\mathrm{e}^{-\frac{d_{1}^{2}}{2}}\right)-\frac{\beta \sigma_{T}^{2}}{\alpha} \mathrm{e}^{\mu_{T}+\frac{1}{2} \sigma_{T}^{2}}\left[N\left(d_{1}\right)-N\left(d_{2}\right)\right] \\
& =-\frac{\beta}{\alpha}\left(\mu_{T}-\ln K+\sigma_{T}^{2}\right) \mathrm{e}^{\mu_{T}+\frac{1}{2} \sigma_{T}^{2}}\left[N\left(d_{1}\right)-N\left(d_{2}\right)\right] \\
& -\frac{\beta}{\alpha} \frac{\sigma_{T}}{\sqrt{2 \pi}} \mathrm{e}^{\mu_{T}+\frac{1}{2} \sigma_{T}^{2}}\left(\mathrm{e}^{-\frac{d_{2}^{2}}{2}}-\mathrm{e}^{-\frac{d_{1}^{2}}{2}}\right),
\end{aligned}
$$

and

$$
\begin{aligned}
I_{3}= & \int_{(1+\alpha) K}^{+\infty}\left[\left(1-\frac{\beta}{\alpha} \ln (1+\alpha)\right) S_{T}+K(\beta-1)\right] f\left(S_{T}\right) \mathrm{d} S_{T} \\
= & \left(1-\frac{\beta}{\alpha} \ln (1+\alpha)\right) \int_{\ln (1+\alpha) K}^{+\infty} \mathrm{e}^{y} f\left(\mathrm{e}^{y}\right) \mathrm{e}^{y} \mathrm{~d} y+K(\beta-1) \int_{\ln (1+\alpha) K}^{+\infty} f\left(\mathrm{e}^{y}\right) \mathrm{e}^{y} \mathrm{~d} y \\
= & \left(1-\frac{\beta}{\alpha} \ln (1+\alpha)\right) \frac{1}{\sqrt{2 \pi} \sigma_{T}} \int_{\ln (1+\alpha) K}^{+\infty} \mathrm{e}^{y} \mathrm{e}^{-\frac{1}{2}\left(\frac{y-\mu_{T}}{\sigma_{T}}\right)^{2}} \mathrm{~d} y \\
& +K(\beta-1) \frac{1}{\sqrt{2 \pi} \sigma_{T}} \int_{\ln (1+\alpha) K}^{+\infty} \mathrm{e}^{-\frac{1}{2}\left(\frac{y-\mu_{T}}{\sigma_{T}}\right)^{2}} \mathrm{~d} y \\
= & \left(1-\frac{\beta}{\alpha} \ln (1+\alpha)\right) \mathrm{e}^{\mu_{T}+\frac{1}{2} \sigma_{T}^{2}} N\left(\frac{\mu_{T}+\sigma_{T}^{2}-\ln (1+\alpha) K}{\sigma_{T}}\right) \\
& +K(\beta-1) N\left(\frac{\mu_{T}-\ln (1+\alpha) K}{\sigma_{T}}\right) \\
= & \left(1-\frac{\beta}{\alpha} \ln (1+\alpha)\right) \mathrm{e}^{\mu_{T}+\frac{1}{2} \sigma_{T}^{2}} N\left(-d_{1}\right)+K(\beta-1) N\left(-d_{3}\right) .
\end{aligned}
$$


Thus, from Formula (3.20) to (3.32), the new option price at time 0 under the stochastic interest rate can be given by

$$
\begin{aligned}
C= & A(0, T) \mathrm{e}^{-r_{0} B(0, T)}\left(1+\frac{\beta}{\alpha}\left(1-\mu_{T}+\ln K-\sigma_{T}^{2}\right)\right) \mathrm{e}^{\mu_{T}+\frac{1}{2} \sigma_{T}^{2}}\left[N\left(d_{1}\right)-N\left(d_{2}\right)\right] \\
& -A(0, T) \mathrm{e}^{-r_{0} B(0, T)}\left(1+\frac{\beta}{\alpha}\right) K\left[N\left(d_{3}\right)-N\left(d_{4}\right)\right] \\
& -A(0, T) \mathrm{e}^{-r_{0} B(0, T)} \frac{\beta}{\alpha} \frac{\sigma_{T}}{\sqrt{2 \pi}} \mathrm{e}^{\mu_{T}+\frac{1}{2} \sigma_{T}^{2}}\left(\mathrm{e}^{-\frac{d_{2}^{2}}{2}}-\mathrm{e}^{-\frac{d_{1}^{2}}{2}}\right) \\
& +A(0, T) \mathrm{e}^{-r_{0} B(0, T)}\left(1-\frac{\beta}{\alpha} \ln (1+\alpha)\right) \mathrm{e}^{\mu_{T}+\frac{1}{2} \sigma_{T}^{2}} N\left(-d_{1}\right) \\
& +A(0, T) \mathrm{e}^{-r_{0} B(0, T)} K(\beta-1) N\left(-d_{3}\right)
\end{aligned}
$$

where

$$
\begin{aligned}
& A(0, T)=\exp \left\{[B(0, T)-T]\left(\frac{\theta}{a}-\frac{\sigma_{2}^{2}}{2 a^{2}}\right)-\frac{\sigma_{2}^{2} B(0, T)^{2}}{4 a}\right\}, \\
& B(0, T)=\frac{1-\mathrm{e}^{-a T}}{a}, \\
& d_{1}=\frac{\ln (1+\alpha) K-\mu_{T}-\sigma_{T}^{2}}{\sigma_{T}}, \\
& d_{2}=\frac{\ln K-\mu_{T}-\sigma_{T}^{2}}{\sigma_{T}}, \\
& d_{3}=\frac{\ln (1+\alpha) K-\mu_{T}}{\sigma_{T}}, \\
& d_{4}=\frac{\ln K-\mu_{T}}{\sigma_{T}}, \\
& \mu_{T}=\ln S_{0}-\frac{\sigma_{1}^{2}}{2} T-r_{0} B(0, T)+\left(\frac{\theta}{a}-\frac{\sigma_{2}^{2}}{a^{2}}\right)[T-B(0, T)]+\frac{\sigma_{2}^{2}}{2 a} B(0, T)^{2}, \\
& \sigma_{T}^{2}=\frac{\sigma_{2}^{2}}{a^{2}}\left[T-2 B(0, T)+\frac{1-\mathrm{e}^{-2 a T}}{2 a}\right]+\sigma_{1}^{2} T .
\end{aligned}
$$

It is obviously that the Formula (3.34) is similar to the option pricing formula of Wang when the interest rate is a constant and $P(0, T), \mu_{T}, \sigma_{T}$ respectively substituted by $\mathrm{e}^{-r T}, \ln S_{0}+\left(\mu-\frac{\sigma_{1}^{2}}{2}\right) T, \sigma_{1} \sqrt{T}$, where $r$ is the riskiness interest rate, $\mu$ expected rate of return of stock price.

Wang [7] gives the following formula for the option pricing based on the designed linear investment strategy:

$$
\begin{aligned}
V(t, S, \alpha, \beta)= & M-H \sigma \sqrt{T-t})\left[N\left(d_{2}\right)-N\left(d_{1}\right)\right] \\
& -\left(1+\frac{\beta}{\alpha}\right) K \mathrm{e}^{-r(T-t)}\left[N\left(d_{4}\right)-N\left(d_{3}\right)\right]+Q S N\left(d_{2}\right) \\
& -(1-\beta) K \mathrm{e}^{-r(T-t)} N\left(-d_{4}\right)-\frac{H}{\sqrt{2 \pi}}\left(\mathrm{e}^{-\frac{d_{1}^{2}}{2}}-\mathrm{e}^{-\frac{d_{2}^{2}}{2}}\right)
\end{aligned}
$$


where

$$
\begin{aligned}
& M=S\left(1+\frac{\beta}{\alpha}\right)-\frac{\beta S}{\alpha}\left[\ln \frac{S}{K}+(T-t)\left(r-\frac{\sigma^{2}}{2}\right)\right] \\
& H=\frac{\beta S}{\alpha} \sigma \sqrt{T-t} \\
& Q=1-\frac{\beta}{\alpha} \ln (1+\alpha) \\
& d_{1}=\frac{\ln \frac{K}{S}-(T-t)\left(r+\frac{\sigma^{2}}{2}\right)}{\sigma \sqrt{T-t}} \\
& d_{2}=\frac{\ln \frac{(1+\alpha) K}{S}-(T-t)\left(r+\frac{\sigma^{2}}{2}\right)}{\sigma \sqrt{T-t}} \\
& d_{3}=\frac{\ln \frac{K}{S}-(T-t)\left(r-\frac{\sigma^{2}}{2}\right)}{\sigma \sqrt{T-t}} \\
& d_{4}=\frac{\ln \frac{(1+\alpha) K}{S}-(T-t)\left(r-\frac{\sigma^{2}}{2}\right)}{\sigma \sqrt{T-t}}
\end{aligned}
$$

It is easily to obtain that the Formula (3.36) we have calculated is equal to the result of Wang 3.37 when the interest rate is a constant. Therefore, it is can confirm the truth of option pricing under the designed investment strategy we have derived. So that the option price under the designed investment strategy with interest rate has been calculated under the $T$-forward measure. Most important, the zero coupon bond is chosen as the numeraire which simplifies the option pricing problem.

\section{Conclusions}

A new option pricing under the designed investment strategy has been researched by Wang [6] [7]. We discuss this new option price under the assumption that the stochastic interest rate is submitted to Vasicek rate model. The classical method of calculating the option pricing is under the risk-neutral measure to make it and this method is too complicated. However, in fact, changing the measure appropriately will simplify the calculation of option pricing. In this case, we chose the zero coupon bond as the numeraire and discussed the option pricing under the $T$-forward measure. It is proved that calculating the option price under the $T$-forward measure simplifies the issue greatly. Finally, the new option pricing under the designed investment strategy with the stochastic interest rate has been calculated. The result formula is similar to the formula represented by Wang [7] which means the proposed conclusion in this paper is feasible.

Of course, there are many deficiencies in this article. Firstly, option under the 
designed investment strategy can be further discussed. For example, will the investment strategy during the option's valid period influence the other aspects of stock option? Are there any differences with the option pricing under the $T$-forward measure and that under the risk-neutral measure? How do the option prices under the investment strategy change, if the interest rate obeys to CoxCIngersollCRoss model, BlackCDermanCToy model, BlackCKarasinski model or HullCWhite model? Moreover, option pricing with stochastic volatility may provide another story. This consideration is worth to be examined.

\section{Funding}

This work was supported in part by the National Nature Science Foundations of China under Grant No. 61673103 and No.61403248.

\section{References}

[1] Black, F. and Scholes, M. (1973) The Pricing of Options and Corporate Liabilities. Journal of Political Economy, 81, 637-659. https://doi.org/10.1086/260062

[2] Merton, R.C. (1973) Theory of Rational Option Pricing. Bell Journal of Economics and Management Science, 4, 141-183. https://doi.org/10.2307/3003143

[3] Cox, J.C. and Ross, S.A. (1976) The Valuation of Options for Alternative Stochastic Processes. Journal of Financial Economics, 3, 145-166. https://doi.org/10.1016/0304-405X(76)90023-4

[4] Cox, J.C., Ross, S.A. and Rubinstein, M. (1985) Option Pricing: a Simplified Approach. Journal of Financial Economics, 7, 229-263. https://doi.org/10.1016/0304-405X(79)90015-1

[5] Buraschi, A. and Dumas, B. (2001) The Forward Valuations of Compound Options. Journal of Derivatives, 9, 8-17. https://doi.org/10.3905/jod.2001.319165

[6] Wang, X.F. and Wang, L. (2007) Study on the Black-Scholes Stock Put Option Model Based on Dynamic Investment Strategy. International Journal of Innovative Computing, Information and Control, 3, 1755-1780.

[7] Wang, X.F. and Wang, L. (2009) Study on Black-Scholes Option Pricing Model Based on General Linear Investment Strategy. International Journal of Innovative Computing, Information and Control, 5, 2169-2188.

[8] Yan, J.Z., Feng, C.H., Luo, D.J. and Liu, Q. (2015) Study on the Call Option Pricing Model under the Nonlinear Dynamic Investment Strategy. Journal of China West Normal University (Natural Sciences), 36, 339-344. (In Chinese)

[9] Vasicek, O. (1977) An Equilibrium Characterization of the Term Structure. Journal of Financial Economics, 5, 177-188. https://doi.org/10.1016/0304-405X(77)90016-2

[10] Harrison, J.M. and Kreps, D.M. (1979) Martingales and Arbitrage in Multiperiod Securities Markets. Journal of Economic Theory, 20, 381-408. https://doi.org/10.1016/0022-0531(79)90043-7

[11] Harrison, J.M. and Pliska, S.R. (1981) Martingales and Stochastic Integrals in the Theory of Continuous Trading. Stochastic Processes and Their Applications, 11, 215-260. https://doi.org/10.1016/0304-4149(81)90026-0

[12] Harrison, J.M. and Pliska, S.R. (1983) A Stochastic Calculus Model of Continuous Trading: Complete Markets. Stochastic Processes and Their Applications, 15, 313-316. https://doi.org/10.1016/0304-4149(83)90038-8 
[13] Heath, D., Jarrow, R. and Morton, A. (1992) Bond Pricing and the Term Structure of Interest Rates: A New Methodology. Econometrica, 60, 77-105. https://doi.org/10.2307/2951677

[14] Geman, H., El Karoui, N. and Rochet, J.C. (1995) Changes of Numeraire, Changes of Probability Measures and Pricing of Options. Journal of Applied Probability, 32, 443-458. https://doi.org/10.1017/S002190020010289X

[15] Musiela, M. and Rutkowski, M. (1998) Martingale Methods in Financial Modeling. Springer, Berlin, 383-416.

[16] Hunt, P. and Kennedy, J. (2000) Financial Derivatives in Theory and Practice. Wiley, Chichester, 19-206.

[17] Jamshidian, F. (1989) An Exact Bond Option Pricing Formula. The Journal of Finance, 44, 205-209. https://doi.org/10.1111/j.1540-6261.1989.tb02413.x

[18] Wang, L.J. and Zhang, S.G. (2003) Pricing the Asian Option under Vasicek Interest Rate. Acta Mathematicae Applicatae Sinica, 26, 467-474. (In Chinese)

[19] Yao, L.G., Wang, X. and Yang, X.Q. (2004) Pricing Asian Option under Vasicek Interest Rate. Natural Science Journal of Xiangtan University, 26, 20-23. (In Chinese)

[20] Xao, B. (2012) European Option Pricing Based on the Stochastica Interest Rate Model. Haibin Engineering University, Haibin, 3. (In Chinese)

[21] Feng, K. (2013) An Empirical Analysis of Option Pricing under Stochastic Interest. Mathemastic and Statistics Central China Normal University, 3-8. (In Chinese)

[22] Cui, X. (2012) Analysis and Simulation of the Option Pricing under Stochastic Interest Rate Model. Shandong University, Jinan, 3-22. (In Chinese)

[23] Brigo, D. and Mercurio, F. (2006) Interest Rate Models-Theory and Practice, with Smile, Inflation and Credit. 2nd Edition, Springer, Berlin, 26-62. 